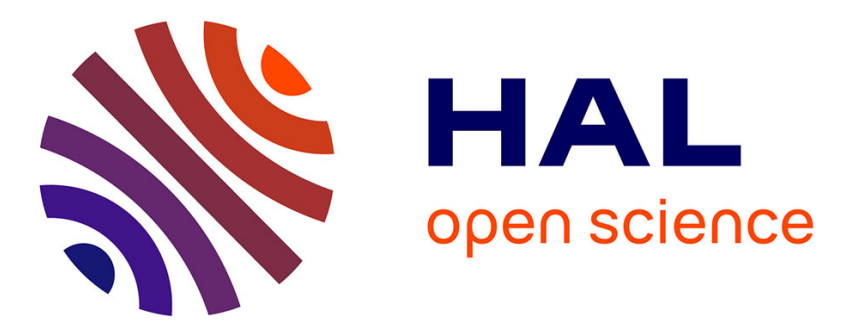

\title{
Comparison of two parametrizations of a turbulence-induced flocculation model through global sensitivity analysis
}

\author{
Régis Cottereau, Fernando A. Rochinha, Alvaro L. G. A. Coutinho
}

\section{To cite this version:}

Régis Cottereau, Fernando A. Rochinha, Alvaro L. G. A. Coutinho. Comparison of two parametrizations of a turbulence-induced flocculation model through global sensitivity analysis. Continental Shelf Research, 2014, 85, pp.85-95. 10.1016/j.csr.2014.07.002 . hal-01068996

\section{HAL Id: hal-01068996 https://hal.science/hal-01068996}

Submitted on 26 Sep 2014

HAL is a multi-disciplinary open access archive for the deposit and dissemination of scientific research documents, whether they are published or not. The documents may come from teaching and research institutions in France or abroad, or from public or private research centers.
L'archive ouverte pluridisciplinaire HAL, est destinée au dépôt et à la diffusion de documents scientifiques de niveau recherche, publiés ou non, émanant des établissements d'enseignement et de recherche français ou étrangers, des laboratoires publics ou privés. 


\title{
Comparison of two parameterizations of a turbulence-induced flocculation model through global sensitivity analysis
}

\author{
R. Cottereau ${ }^{\mathrm{a}, *}$, F. A. Rochinha ${ }^{\mathrm{b}}$, A. L. G. A. Coutinho ${ }^{\mathrm{c}}$ \\ ${ }^{a}$ Laboratoire MSSMat, École Centrale Paris, CNRS, Grande voie des vignes, F-92295 \\ Châtenay-Malabry, France \\ ${ }^{b}$ Mechanical Engineering Department, COPPE, Federal University of Rio de Janeiro, \\ Rio de Janeiro, Brazil \\ ${ }^{c}$ High Performance Computing Center $\&$ Civil Engineering Department, COPPE, \\ Federal University of Rio de Janeiro, Rio de Janeiro, Brazil
}

\begin{abstract}
This paper describes a global sensitivity analysis of a fractal-based turbulenceinduced flocculation model. The quantities of interest in this analysis are related to the floc diameters in two different configurations. The input parameters with which the sensitivity analyses are performed are the floc aggregation and breakup parameters, the fractal dimension and the diameter of the primary particles. Two related versions of the flocculation model are considered, evenly encountered in the literature: (i) using a dimensional floc breakup parameter, and (ii) using a non-dimensional floc breakup parameter. The main results of the sensitivity analyses are that only two parameters of model (ii) are significant (aggregation and breakup parameters) and that the relationships between parameter and quantity of interest remain simple. Contrarily, with model (i), all parameters have to be considered. When
\end{abstract}

*regis.cottereau@ecp.fr 
identifying model parameters based on measures of floc diameters, this analysis hence suggests the use of model (ii) rather than (i). Further, improved models of the fractal dimension do not seem to be required when using the non-dimensional model (ii).

Keywords: Flocculation, Sensitivity Analysis, Analysis of Variance, Gravity Currents, Turbidity Currents.

\section{Introduction}

Flocculation is the property of small cohesive particles to form so-called flocs under the action of electrostatical forces. The flocs are larger groups of particles whose influence can be dramatic in many physical problems, such as for turbidity currents (Thomas et al., 1999; Geyer et al., 2004; Jarvis et al., 2005; Meiburg and Kneller, 2010). The study and modeling of these turbidity currents (Cantero et al., 2006; Elias et al., 2008; Hiester et al., 2011; NasrAzadani et al., 2013; Guerra et al., 2013; Espath et al., 2014) is particularly difficult because the particle concentration, and hence the driving buoyancy force, fluctuates with time and position along the current, as well as in the vertical direction. The influence of flocculation is large in that case because the settling velocity of mud flocs can be a few orders of magnitude larger than that of the primary particles, so that its effect on particle concentration is very important.

The flocculation process is influenced by sediment size, sediment concentration, turbulence intensity, temperature, and the presence of organic matter. It is governed by three processes that drive particles to collide: (i) brownian motion, (ii) differential settling of fast particles overtaking slower 
particles, and (iii) turbulent motion. In estuaries and coastal regions, the first is arguably negligible (Winterwerp, 1998; Cuthbertson et al., 2010). Concerning differential settling, which is likewise often disregarded, a model is described in Lick et al. (1993) and experimental results are reported in Cuthbertson et al. (2010). About the effect of turbulent motion, more widely studied, aggregation theory suggests that aggregation rates increase with the turbulence intensity, due to the increased number of inter-particle encounters. However, the same turbulent motions may generate shear stresses that can limit the growth rate through floc disruption and break-up (disaggregation) processes.

Following the pioneering work of von Smoluchowski (1917), who derived the time variation of the concentration of a mono-disperse suspension due to Brownian diffusion, most studies of flocculation in turbidity currents have considered population balance equations along with empirical models of creation and disruption of flocs of different sizes (see for instance Thomas et al. (1999); Son and Hsu (2008); Chao and Jia (2011) and references therein). Many different population balance equations based on Lagrangian or Eulerian formulations have been proposed (Friedlander, 1957; O'Melia, 1980; Spicer and Pratsinis, 1996; Serra and Casamitjana, 1998; McAnally and Mehta, 2002; Rahmani et al., 2004), each taking into account different processes. Winterwerp (1998) developed a model for the turbulence-induced flocculation, a priori aimed at modeling flocculation in estuary and coastal areas. Although initially considering the evolution of flocs of a single characteristic diameter, this model was later extended to the poly-disperse case (Xu et al., 2008). Some authors also extended the model to fractal dimensions 
dependent on the floc size (Maggi and Winterwerp, 2004; Khelifa and Hill, 2006; Vahedi and Gorczyca, 2011). All these models rely on several parameters that have to be identified in experiments, and several parameterizations sometimes exist for the same model.

The choice of parameterization influences the identifiability of models. For instance, if a given quantity of interest (QoI) does not depend on one of the model parameters, it means that this parameter can be chosen at will without degrading the estimation of that QoI. Conversely, the sensitivity of a QoI with respect to the parameters indicates the parameters that will be polluted by noise and those that will be identified with more confidence. The reason why a given QoI might be more or less sensitive to one or another parameter is due, among other things, (i) to the particular form of the model (the relation between parameters and QoI might be very non-linear), and (ii) to differences of orders of magnitudes between the parameters. Sensitivity analysis (Saltelli et al., 2000) is the study of these dependencies between model parameters and QoIs. It hence allows to identify parameters that should be the focus of more attention.

In the context of flocculation models, such sensitivity analyses have been performed for two QoIs (floc diameter and settling velocity) and with respect to some parameters in Khelifa and Hill (2006); Son and Hsu (2008); Maerz et al. (2011). In particular, a major conclusion of the sensitivity study in Maerz et al. (2011) points at the fractal dimension as the most influential parameter on the floc diameter, while its experimental identification is generally difficult (Bushell et al., 2002). Among other things, this conclusion motivates the use of refined models of that fractal dimension. In Mietta 
et al. (2008), the authors presented a sensitivity study with respect to the breakup functions, that is to say with respect to different functional forms of the breakup model. Note that all these analyses remained local, in the sense that the influence of variations of the parameters (one at the time) was observed around one reference model. Conclusions drawn from a local sensitivity analysis (SA) around a given reference might be modified by the consideration of another reference point.

Contrarily to local SA, global SA aims at investigating the functional form of a model rather than the behavior around a particular design point. The objectives of global SA are the same as in local SA, but the influence of the parameters is considered jointly over the entire range of all the parameters. In particular, this means that non-linearities between parameters and QoIs are explicitly considered, while local SA explore linearized models. To the best of our knowledge, this paper presents the first global SA of flocculation models. We concentrate here on Winterwerp's model (Winterwerp, 1998), for a unique characteristic diameter and constant fractal dimension, and perform a Monte-Carlo based global SA (Helton et al., 2006; Saltelli et al., 2008) with respect to four model parameters. Two of the parameters we consider are common to all flocculation models (although sometimes presented in the form of efficiency parameters): the flocculation parameter $k_{A}$ and the breakup parameter $k_{B}$. Two parameters are particular to fractalbased models: the fractal dimension $n_{f}$ and the primary particle diameter $D_{P}$. The QoIs that we consider are all related to floc diameters (average, minima and maxima). This SA will be performed for two versions of the model, equally encountered in the literature: (i) using a dimensional floc 
breakup parameter, and (ii) using a non-dimensional floc breakup parameter. The analysis allows to propose a clear recommendation on the most appropriate parameterization. In particular, it will allow us to reconsider the need for refined models of the fractal dimension parameter.

Section 2 recalls the main components of the two versions of Winterwerp's model, along with their implementation in a coupled system of transport equations and the definitions of equilibrium diameter and time. Section 3 describes a literature survey of ranges of variations of the flocculation parameters and proposes probabilistic descriptions of these parameters for use in the SA. Section 4 describes the SA performed for the equilibrium diameter with the two different parameterizations of Winterwerp's model. Its conclusion is that the non-dimensional model should be used rather than the dimensional one. Section 5 describes the SA performed for a 0D tidal example taken from Maerz et al. (2011) that confirms the previous result. Finally, some conclusions are presented in Section 6.

\section{Turbulent flocculation model}

In this section, which is mostly based on Winterwerp's original paper (Winterwerp, 1998), we recall the flocculation model that will be considered throughout. It is based on the hypothesis of fractal behavior of the floc size. It proposes a flocculation source term in a population balance equation for the number of flocs per unit volume $N$.

\subsection{Self-similiar flocculation model}

In this model, the aggregates are treated as self-similar entities (Kranenburg, 1994). The model therefore assumes that the flocs are composed of an 
ensemble of so-called primary particles, with diameter $D_{P}$, which bond in a very specific way. This assembly process induces a relation between the mass concentration $c$, the number concentration $N$ and the mass of one primary particle $m_{P}$

$$
c=m_{P} N\left(\frac{D}{D_{P}}\right)^{n_{f}}, \quad N=\frac{c}{m_{P}}\left(\frac{D_{P}}{D}\right)^{n_{f}}, \quad D=D_{P}\left(\frac{c}{m_{P} N}\right)^{1 / n_{f}}
$$

where $n_{f}$ is called the fractal dimension. Fractal dimension and primary particle diameter are two parameters that have to be identified through experiments. Typical values will be discussed in Sec. 3.2.

The flocculation model proposed in Winterwerp (1998) combines linearly a literature model for the aggregation (Levich, 1962) and an original model for the breakup of flocs. It describes the evolution of the number of flocs as:

$$
\delta N=-k_{A} \frac{m_{P}}{\rho_{s}} G\left(\frac{D}{D_{P}}\right)^{3} N^{2}+k_{B} \sqrt{\frac{\mu}{F_{y}}} G^{3 / 2} D\left(\frac{D}{D_{P}}-1\right)^{3-n_{f}} N,
$$

where $\rho_{s}$ is the density of particles, $k_{A}$ is a dimensionless flocculation coefficient, $k_{B}$ is a dimensionless floc breakup parameter, $F_{y}$ is the yield strength of flocs, and $\mu$ is the dynamic viscosity of the fluid. Note that the model presented here is slightly simplified from the original article (Winterwerp, 1998). In particular, processes of deposition and erosion, which are taken into account through additional parameters, are here disregarded.

\subsection{Coupled system of transport equation}

The previous equation describes only the creation and destruction of flocs through flocculation. The space and time evolution of flocs is controlled by two coupled balance equations, for which the above model acts as a source term. Assuming that the flocs are both convected by the flow of carrying 
fluid with a velocity $\mathbf{w}$, and also naturally settle with a (vertical) velocity $\mathbf{w}_{s}$, this transport equation is:

$$
\frac{\partial N}{\partial t}+\nabla \cdot\left(\left(\mathbf{w}-\mathbf{w}_{s}\right) N-K_{N} \nabla N\right)=\delta N
$$

where $K_{N}$ is a diffusion tensor (assumed given and diagonal). The settling velocity of the flocs depends on their size. Assuming that the flocs are spherical, and at low Reynolds number, the settling velocity can be modeled as:

$$
\begin{aligned}
\mathbf{w}_{s}(D) & =-\frac{\left(\rho_{s}-\rho_{w}\right) g D_{P}^{2}}{18 \mu}\left(\frac{D}{D_{P}}\right)^{n_{f}-1} \mathbf{e}_{z} \\
& =-\frac{\left(\rho_{s}-\rho_{w}\right) g D_{P}^{2}}{18 \mu}\left(\frac{c}{m_{P} N}\right)^{1-1 / n_{f}} \mathbf{e}_{z},
\end{aligned}
$$

where $\rho_{w}$ is the density of water, $g$ is the acceleration of gravity, and $\mathbf{e}_{z}$ is the vertical unit vector pointing upward.

Finally the model is closed with a more classical transport equation for the mass concentration $c$ :

$$
\frac{\partial c}{\partial t}+\nabla \cdot\left(\left(\mathbf{w}-\mathbf{w}_{s}\right) c-K_{c} \nabla c\right)=0
$$

where $K_{c}$ is a mixing tensor (assumed given and diagonal). Winterwerp's model therefore consists of a set of two transport equations (3)-(6) for the two unknowns $c$ and $N$. These two transport equations are coupled through both the flocculation source term $\delta N(2)$ and the settling velocity $\mathbf{w}_{s}(4)$. Rewriting the two equations as functions of $c$ and $N$ only, and with the coupling terms on the right-hand sides, yields:

$$
\frac{\partial c}{\partial t}+\nabla \cdot\left(\mathbf{w} c-K_{c} \nabla c\right)=w_{P} \frac{\partial}{\partial z}\left(c\left(\frac{c}{m_{P} N}\right)^{1-1 / n f}\right)
$$




$$
\begin{aligned}
& \frac{\partial N}{\partial t}+\nabla \cdot\left(\mathbf{w} N-K_{N} \nabla N\right)= \\
& w_{P} \frac{\partial}{\partial z}\left(\frac{c}{m_{P}}\left(\frac{m_{P} N}{c}\right)^{1 / n f}\right)-q_{A} N^{2}\left(\frac{c}{m_{P} N}\right)^{3 / n_{f}} \\
& +q_{B} N\left(\frac{c}{m_{P} N}\right)^{1 / n_{f}}\left(\left(\frac{c}{m_{P} N}\right)^{1 / n_{f}}-1\right)^{3-n_{f}},
\end{aligned}
$$

where we have introduced a volume flux of floc aggregation $q_{A}=k_{A} m_{P} G / \rho_{s}$, a number flux of floc breakup $q_{B}=k_{B} D_{P} G \sqrt{\mu G / F_{y}}$ and the settling velocity of a primary particle (assumed spherical) $w_{P}=\left(\rho_{s}-\rho_{w}\right) g D_{P}^{2} /(18 \mu)$. Boundary conditions for that transport model can be taken (for example) as $\left(\mathbf{w} c+K_{c} \nabla c\right) \cdot \mathbf{e}_{z}=0$ and $\left(\mathbf{w} N+K_{N} \nabla N\right) \cdot \mathbf{e}_{z}=0$ at the free surface and on the bottom (assuming there is no erosion or deposition). The above system of equations can be approximated by numerical techniques (see Section 5).

\subsection{Stationary regime}

Before considering the solution of this coupled system of transport equations, it is interesting to discuss the stationary situation in which flocs are created and destroyed at the same rate, so that the floc population and size remain constant. Considering $\delta N=0$ in Eq. (2) yields the following implicit definition for the floc equilibrium diameter $D_{\infty}$ :

$$
D_{\infty}\left(1-\frac{D_{P}}{D_{\infty}}\right)^{3-n_{f}}=\frac{k_{A} c}{k_{B} \rho_{s}} \sqrt{\frac{F_{y}}{\mu G}} .
$$

In the limit $D_{\infty}>>D_{P}$, which is often realized in practice, one obtains the approximation

$$
D_{\infty} \approx\left(3-n_{f}\right) D_{P}+\frac{k_{A} c}{k_{B} \rho_{s}} \sqrt{\frac{F_{y}}{\mu G}} .
$$


We will check in Sec. 4 the accuracy of that approximation. One can also define a characteristic time scale when $D_{\infty}>>D_{P}$ as:

$$
T_{\infty}=\frac{n_{f}}{k_{B} G} \sqrt{\frac{F_{y}}{\mu G}} \frac{1}{D_{\infty}}\left(\frac{D_{P}}{D_{\infty}}\right)^{3-n_{f}}
$$

This characteristic flocculation time represents the order of magnitude of the time required to reach the equilibrium diameter $D_{\infty}$ when the system is out of equilibrium. These two quantities $D_{\infty}$ and $T_{\infty}$ are useful to characterize grossly the flocculation regimes, depending on the driving phenomena (see Section 5 for an example in a tidal-controlled cyclic flocculation).

\subsection{Alternative parameterization}

Finally, it is important to note that another parameterization is used as

often in the literature as the one that was already presented. Instead of defining a dimensionless floc breakup parameter, this alternative parameterization is based on a floc breakup parameter $k_{B}^{\prime}=k_{B} \sqrt{\left(\mu / F_{y}\right)} /\left(n_{f} D_{P}^{3-n_{f}}\right)$. Although it might appear as an unimportant change of variables, it may have a very important impact on the identifiability of the different parameters of the model. This will be discussed in Section 4 .

When considering this parameterization, the equilibrium diameter is then given by

$$
D_{\infty}\left(1-\frac{D_{P}}{D_{\infty}}\right)^{3-n_{f}}=\frac{k_{A} c}{k_{B}^{\prime} n_{f} \rho_{s} D_{P}^{3-n_{f}} \sqrt{G}},
$$

and, in the limit $D_{\infty}>>D_{P}$,

$$
D_{\infty} \approx\left(3-n_{f}\right) D_{P}+\frac{k_{A} c}{k_{B}^{\prime} n_{f} \rho_{s} D_{P}^{3-n_{f}} \sqrt{G}} .
$$

The characteristic time for the alternative parameterization is

$$
T_{\infty}=\frac{1}{k_{B}^{\prime} G^{3 / 2} D_{\infty}^{4-n_{f}}} .
$$


It should be noted that the dimensional and non-dimensional parameters are perfectly equivalent. Given the set of parameters including the nondimensional $k_{B}$, one can define in a unique and equivalent way the dimensional parameter $k_{B}^{\prime}$. Likewise, given the set of parameters including the dimensional $k_{B}^{\prime}$, one can define in a unique and equivalent way the nondimensional parameter $k_{B}$. However, this choice of parameterization modifies drastically the relationship between the parameters and the quantities of interest (the equilibrium diameter for instance). This is particularly true here, where the relationship between $k_{B}^{\prime}$ and $k_{B}$ involves two other parameters $n_{f}$ and $D_{P}$. Among other things this means that the sensitivity to errors in the parameters might be modified for the two cases.

\section{Description of the flocculation parameters}

SA refers to the determination of the contributions of individual uncertain inputs to the uncertainty in analysis results. A number of approaches have been developed, including differential analysis, response surface methodologies, Monte Carlo analyses, and variance decomposition methods. We will concentrate here on Monte Carlo analyses (Helton et al., 2006). The objective of the paper is to study the sensitivity of some QoIs with respect to the parameters $k_{A}, k_{B}, n_{f}$ and $D_{P}$. The first step of such a SA consists in choosing appropriate probability density functions for the input parameters. The approach that is chosen here consists in considering a database of experimentally-identified values of these parameters reported in the literature. From that database, average and variance are selected, and the distribution is chosen based on the Maximum Entropy Principle (Shannon, 
1948; Jaynes, 1957), the known bounds on the parameters and the given average and variance. Examples of construction of stochastic models using this method can be found in Udwadia (1989); Soize (2000); Cottereau et al. (2007); Adhikari and Friswell (2007).

\subsection{Literature survey of flocculation parameters}

Values of the four parameters of interest found in the literature are reported in Table 1. In most of the corresponding papers, the authors do not experimentally identify the value of $n_{f}$, but rather choose it equal to 2 before concentrating on identifying the values of $k_{A}$ and $k_{B}$. The choice of $n_{f}=2$ has the advantage that the formulas of the flocculation model simplify so that the identification becomes simpler.

Table 1: Literature values of flocculation parameters.

\begin{tabular}{|c|c|c|c|c|c|}
\hline Reference & $k_{A}$ & $k_{B}$ & $n_{f}$ \\
{$[-]$} & {$[-]$} & $\begin{array}{c}D_{P} \\
{[-]}\end{array}$ & Data from \\
{$[\mu \mathrm{m}]$} & \\
\hline \hline Winterwerp (1998) & 0.31 & $3.5 \times 10^{-5}$ & 2 & 4 & \\
Son and Hsu (2008) & 0.33 & $1.1 \times 10^{-5}$ & 2 & 15 & Manning and Dyer (1999) \\
Son and Hsu (2008) & 0.3 & $1.4 \times 10^{-5}$ & 2 & 15 & Manning and Dyer (1999) \\
Son and Hsu (2008) & 0.008 & $4.3 \times 10^{-5}$ & 2 & 15 & Biggs and Lant (2000) \\
Son and Hsu (2008) & 1.02 & $0.4 \times 10^{-5}$ & 2 & 5 & Bouyer et al. (2004) \\
Mietta et al. (2008) & 0.46 & $24.0 \times 10^{-5}$ & 2 & 6 & Maggi et al. (2002) \\
Maerz et al. (2011) & 0.18 & $2.9 \times 10^{-5}$ & 1.9 & 4 & \\
Maerz et al. (2011) & 0.4 & $11.5 \times 10^{-5}$ & 1.9 & 4 & \\
\hline
\end{tabular}


However, this (almost) constant value of $n_{f}=2$ throughout the reported experiments does not reflect the variability that seems to arise in other sets of experiments. We therefore performed a larger study of the literature, concentrating on obtaining a relevant database for the fractal dimension. The values are reported in Table 2. Note that it is possible to derive values of the fractal dimension based on theoretical considerations of aggregation mechanisms at the molecular scale (see for instance Meakin (1984); Thouy and Jullien (1994); Vahedi and Gorczyca (2012)). However, these results were not used here, because of a lack of knowledge about these mechanisms for the situation we are considering.

Table 2: Ranges of the fractal dimension reported in the literature.

\begin{tabular}{|c|c|c|}
\hline Ref. & $n_{f}[-]$ & Data from \\
\hline \hline Hsu et al. (2007) & 2.15 & \\
Son and Hsu (2008) & {$[1.35-2.3]$} & Bouyer et al. (2004) \\
Vahedi and Gorczyca (2012) & {$[2.55-2.99]$} & \\
Maerz et al. (2011) & {$[1.5-2.4]$} & several databases \\
Khelifa and Hill (2006) & {$[2-3]$} & several databases \\
\hline
\end{tabular}

Finally, we consider the alternative parameterization described at the end of Section 2 and present literature values for the dimensional floc breakup coefficient $k_{B}^{\prime}$ in Table 3 . In this table, the values of $k_{B}^{\prime}$ are reported with the corresponding values of $n_{f}, D_{P}, F_{y}$ and $\mu$ because these values are required to go from $k_{B}$ to and from $k_{B}^{\prime}$. Note that, in the papers reported in the left column of Tables 1 and 3 , it was either $k_{B}$ or $k_{B}^{\prime}$ that was originally used. 
When $k_{B}$ was given in the original paper, we computed the corresponding $k_{B}^{\prime}$ and reported it in Table 3 . When $k_{B}^{\prime}$ was given in the original paper, we computed the corresponding $k_{B}$ and reported it in Table 1.

Table 3: Literature values for the alternative definition of the floc breakup parameter. See Table 1 for the origin of the data.

\begin{tabular}{|c|c|c|c|c|c|}
\hline Reference & $\begin{array}{c}k_{B}^{\prime} \\
{\left[\mathrm{s}^{1 / 2} / \mathrm{m}^{2}\right]}\end{array}$ & $\begin{array}{c}n_{f} \\
{[-]}\end{array}$ & $\begin{array}{c}D_{P} \\
{[\mu \mathrm{m}]}\end{array}$ & $\begin{array}{c}F_{y} \\
{\left[\mathrm{~kg} \cdot \mathrm{m} / \mathrm{s}^{2}\right]}\end{array}$ & $\begin{array}{c}\mu \\
{[\mathrm{kg} / \mathrm{m} / \mathrm{s}]}\end{array}$ \\
\hline \hline Winterwerp (1998) & $14 \times 10^{3}$ & 2 & 4 & $10^{-10}$ & $10^{-3}$ \\
Son and Hsu (2008) & $1.2 \times 10^{3}$ & 2 & 15 & $10^{-10}$ & $10^{-3}$ \\
Son and Hsu (2008) & $1.5 \times 10^{3}$ & 2 & 15 & $10^{-10}$ & $10^{-3}$ \\
Son and Hsu (2008) & $4.6 \times 10^{3}$ & 2 & 15 & $10^{-10}$ & $10^{-3}$ \\
Son and Hsu (2008) & $1.2 \times 10^{3}$ & 2 & 5 & $10^{-10}$ & $10^{-3}$ \\
Mietta et al. (2008) & $63.2 \times 10^{3}$ & 2 & 6 & $10^{-10}$ & $10^{-3}$ \\
Maerz et al. (2011) & $12 \times 10^{3}$ & 1.9 & 4 & $10^{-10}$ & $10^{-3}$ \\
Maerz et al. (2011) & $48 \times 10^{3}$ & 1.9 & 4 & $10^{-10}$ & $10^{-3}$ \\
\hline
\end{tabular}

\subsection{Statistical description of flocculation parameters}

The number of identification experiments reported in the literature is rather limited. It is hence not reasonable to expect identifying full probability density functions from the databases reported above (see Berry et al. (2012) for a more precise discussion). Rather, we will proceed in three steps: (i) identify only the expectation and variance of the parameters $k_{A}, k_{B}, n_{f}$, $D_{P}$ and $k_{B}^{\prime}$ from the literature values, (ii) identify the form of the firstorder marginal distribution using the Maximum Entropy Principle and the 
known bounds of the parameters, and (iii) identify the cross-correlations between the parameters. The first-order marginal densities are the probability density functions of each random variable considered alone, discarding the potential correlation or higher-order relations between these variables. In this approach, and because of the limited information available in the literature, we only aim at constraining (i) the first-order marginal densities and (ii) cross-correlations.

Concerning the first step, and based on the discussion above, the parameters $k_{A}, k_{B}$ and $D_{P}$ are identified from the values in Table 1 . The fractal dimension $n_{f}$ is identified from the values in both Tables 1 and 2, considering only the middle value for the intervals. Finally, the dimensional floc breakup parameter $k_{B}^{\prime}$ is identified from the values in Table 3. The statistical expectation and variance obtained for these five parameters are reported in Table 4. The unbiased estimates for the expectation and variance are used $\mu_{X}=\sum_{i=1}^{N} x_{i} / N$ and $\sigma_{X}^{2}=\sum_{i=1}^{N}\left(x_{i}-\mu_{X}\right)^{2} /(N-1)$, where the $x_{i}$ are the $N$ realizations of the random variable $X$. The correlation coefficient is computed as $\xi_{X}=\sigma_{X} / \mu_{X}$.

Table 4: Statistical estimates for the flocculation parameters (based on Tables 1 and 3).

\begin{tabular}{|c|c|c|c|c|c|}
\hline & $k_{A}[-]$ & $k_{B}[-]$ & $n_{f}[-]$ & $D_{P}[\mu \mathrm{m}]$ & $k_{B}^{\prime}\left[\mathrm{s}^{1 / 2} / \mathrm{m}^{2}\right]$ \\
\hline Expectation $\mu$ & 0.38 & $6.2 \times 10^{-5}$ & 2.2 & 8.5 & $18.2 \times 10^{3}$ \\
Variance $\sigma^{2}$ & 0.09 & $64.1 \times 10^{-10}$ & 0.15 & 29.4 & $573 \times 10^{6}$ \\
Correlation coefficient $\xi$ & 0.8 & 1.7 & 0.2 & 0.6 & 1.3 \\
\hline
\end{tabular}


We then turn to the choice of the first-order marginal density functions for each of the parameters. The Maximum Entropy Principle (Shannon, 1948; Jaynes, 1957) allows us to select the least informative distribution given a series of constraints. In particular, in our case, we can consider the average and variance identified above as constraints. Further, the parameters have different supports that should be considered as constraints also. By nature, the parameters $k_{A}, k_{B}, D_{P}$, and $k_{B}^{\prime}$ are positive, while the fractal dimension has bounded support $0<n_{f}<3$. Based on these constraints, the Maximum Entropy Principle proposes to use truncated Gaussian distributions for all the parameters (Udwadia, 1989). The first-order marginal distributions for $k_{A}, k_{B}, n_{f}$ and $D_{P}$ are plotted in Fig. 1, with the appropriate choice of expectation and variance.
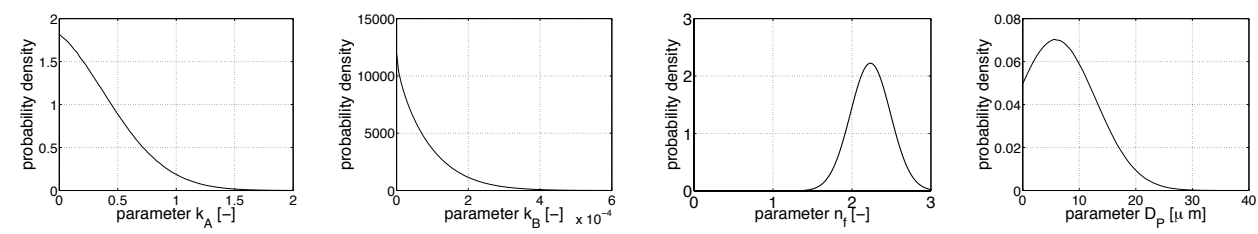

Figure 1: Probability distributions of the flocculation parameters.

Finally, the consideration of appropriate correlation between the input parameters is particularly important in sensitivity analyses. We therefore compute, again using the values from Table 1, the correlation between the four parameters. The unbiased estimate of the correlation is $\sigma_{X X^{\prime}}^{2}=\sum_{i=1}^{N}\left(x_{i}-\mu_{X}\right)\left(x_{i}^{\prime}-\mu_{X}^{\prime}\right) /(N-1)$, where the $x_{i}$ and $x_{i}^{\prime}$ are the $N$ realizations of the random variables $X$ and $X^{\prime}$, respectively. The correlation between the fractal dimension and the other parameters cannot be estimated, because its distribution is determined from a 
different database. In that case, the Maximum Entropy Principle states that the fractal dimension should be considered as independent from the other parameters. The matrix of correlation coefficients $R$ between the different parameters (in the order $k_{A}, k_{B}, n_{f}, D_{P}$ ) is therefore:

$$
R=\left[\begin{array}{cccc}
1 & 0 & 0 & -0.4 \\
0 & 1 & 0 & -0.3 \\
0 & 0 & 1 & 0 \\
-0.4 & -0.3 & 0 & 1
\end{array}\right]
$$

Note that the lack of correlation between the aggregation and breakup parameters $k_{A}$ and $k_{B}$ arises from the data analyzed and has not been assumed as vanishing. We denote, for later use, the non-zero extra-diagonal values of the matrix as $R_{A D}=-0.4$ and $R_{B D}=-0.3$. When considering $k_{B}^{\prime}$ instead of $k_{B}$ (and using the values in Table 3), one obtains $R_{B^{\prime} D}=-0.46$, and the rest of the matrix remains unchanged.

The generation of realizations of the flocculation parameters following the identified first-order marginal laws (Fig. 1), and correlation matrix $R$ is discussed in Appendix A. This concludes the construction of appropriate probability distributions for the flocculation parameters. The next two sections report two sensitivity analyses for different QoIs.

\section{Sensitivity analysis for equilibrium diameter}

We now turn to the first SA. We want to analyze the influence of the flocculation parameters on the equilibrium diameter defined by Eq. (9), and approximated by Eq. (10). At least two reasons make this quantity important in applications: (i) it is directly measurable in identification experiments 
(through direct image analysis, or through diffraction patterns (Bushell et al., 2002)), and (ii) it has a strong influence on the settling velocity, which is another measurable quantity, and one of the most important QoIs for industrial applications.

All the computations in this section are done with $G=10 \mathrm{~s}^{-1}$ and $c=$ $1 \mathrm{~g} / \mathrm{L}$. They were repeated with different pairs of values with no qualitative influence on the results (except for very large values of $G$ or very small values of $c$ that induce $\left.D_{\infty} \approx D_{P}\right)$. The other numerical parameters are $F_{y}=10^{-10} \mathrm{~kg} \cdot \mathrm{m} / \mathrm{s}^{2}, \mu=10^{-3} \mathrm{~kg} / \mathrm{m} / \mathrm{s}$ and $\rho_{s}=2650 \mathrm{~kg} / \mathrm{m}^{3}$.

\section{1. $1 D$ and 2D histograms}

We first propagate the probability laws of $k_{A}, k_{B}, n_{f}$ and $D_{P}$ through the formula of Eq. (9). We use the Monte Carlo approach, with $10^{7}$ samples and approximate the solution of Eq. (9) for each sample using the bisection

method. We obtain an approximation of the first-order marginal density of the equilibrium diameter, that is represented in Figure 2(a). Note that this histogram is obtained considering homogeneous intervals in log-scale. With that choice, log-normal variables have densities that look gaussian. This choice makes visualization easier for distributions considered here. It will be followed in this paper for all variables, except the fractal dimension $n_{f}$, for which homogeneous intervals will be considered in true scale. The probability density obtained for the approximation of the equilibrium diameter given by Eq. (10) is also shown in Figure 2(a). As expected, the true value and its approximation only differ when the equilibrium diameter $D_{\infty}$ is of the same order of magnitude as the primary diameter $D_{P}$, in which case the approximation formula is not valid. 


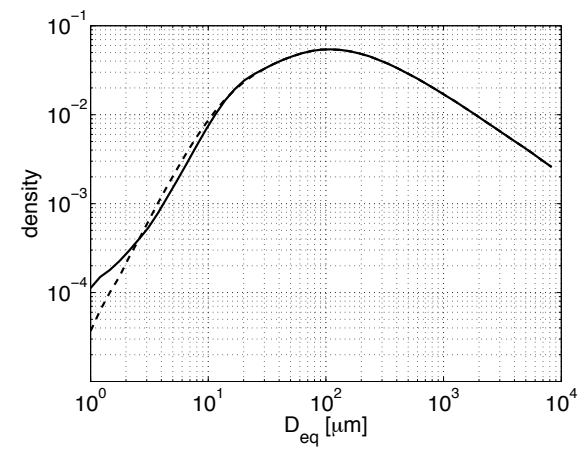

(a) with non-dimensional $k_{B}$

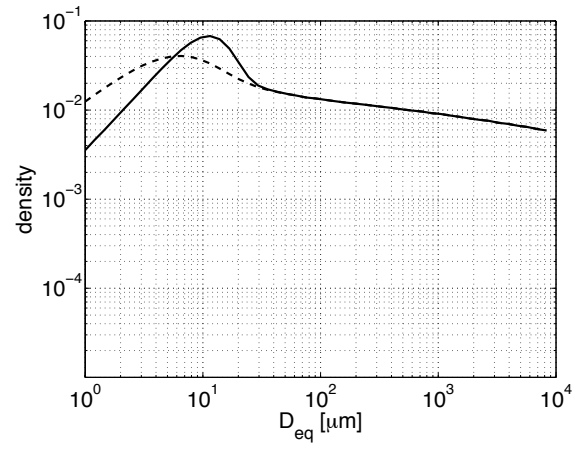

(b) with dimensional $k_{B}^{\prime}$

Figure 2: Probability density function of the equilibrium diameter $D_{\infty}$ given by Eq. (9) (solid line), and of its approximation, given at Eq. (10) (dashed line).

We plot second-order marginal densities between the QoI and each of the input parameters in turn on Figure 3. As before, densities are estimated for $k_{A}, k_{B}, D_{P}$ and $D_{\infty}$ using intervals homogeneous in log-scale, while the density with respect to $n_{f}$ is estimated using homogeneous intervals in true scale. Visually, it seems that $D_{\infty}$ is uncorrelated with $n_{f}$ as the secondorder marginal density appears as a tensorization of the first-order marginal densities. The particle diameter seems only slightly correlated while the two flocculation parameters $k_{A}$ and $k_{B}$ seem to be more correlated.

\subsection{Sensitivity indices}

We now turn to quantitative measures of sensitivity. Correlation provides a measure of the strength of the linear relationship between the output $D_{\infty}$ and one of the input parameters, one after the other. The Pearson correlation 

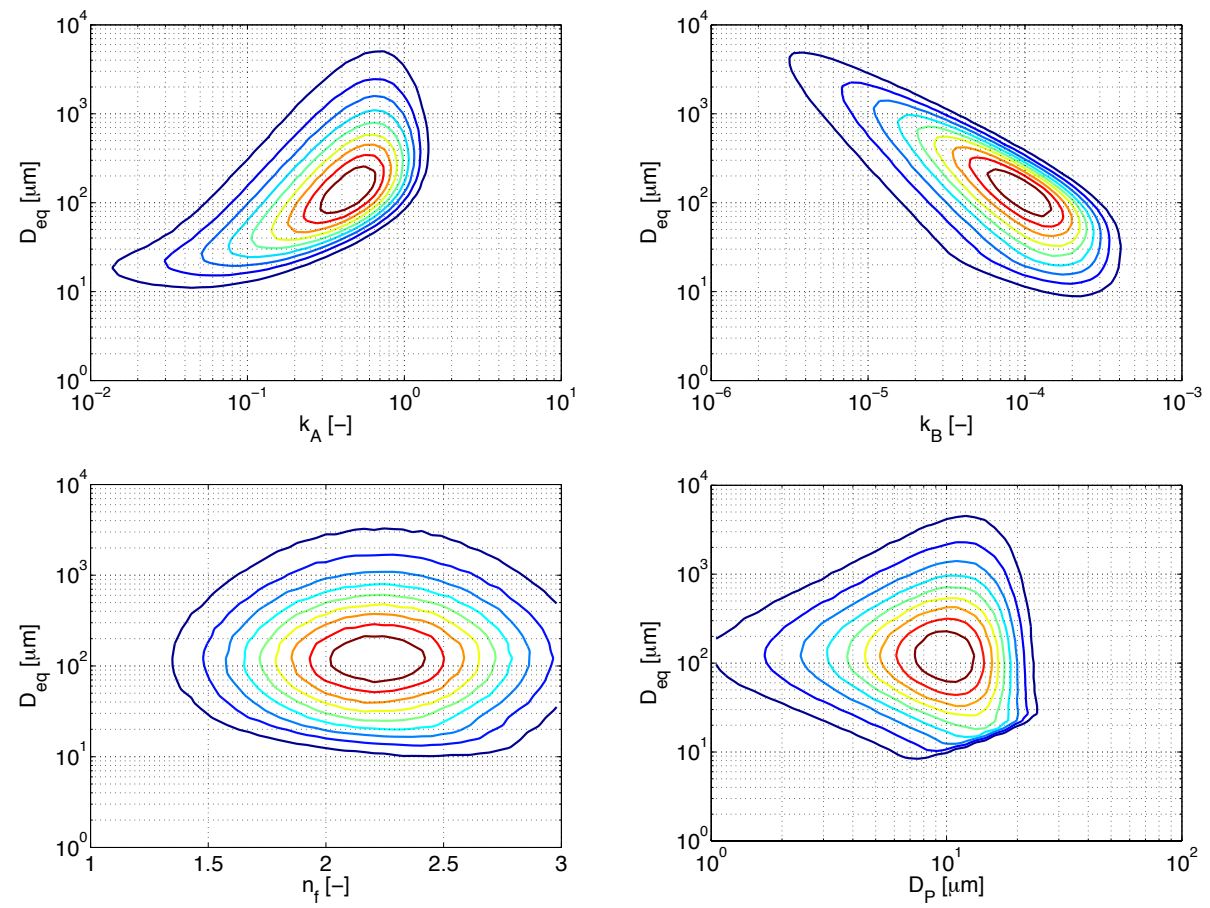

Figure 3: Second-order marginal densities of equilibrium diameter $D_{\infty}$ (considering the parameterization with non-dimensional $k_{B}$ ) with each of the input parameters. The contour lines correspond to the successive $10 \%$ quantiles. 
coefficient between $D_{\infty}$ and a parameter $X$ is defined by:

$$
C C_{X}=\frac{1}{\sigma_{\infty} \sigma_{X}} \sum_{i=1}^{N}\left(D_{\infty, i}-\mu_{\infty}\right)\left(x_{i}-\mu_{X}\right),
$$

where $\mu_{\infty}=\sum_{i=1}^{N} D_{\infty, i} / N$ and $\sigma_{\infty}^{2}=\sum_{i=1}^{N}\left(D_{\infty, i}-\mu_{\infty}\right)^{2} /(N-1), D_{\infty, i}$ are the samples of the equilibrium diameter and $N=10^{7}$ in that case. A vanishing correlation only indicates the absence of a linear relation between $D_{\infty}$ and the considered parameter. It does not preclude the existence of a well-defined nonlinear relationship between them. Values of the Pearson correlation coefficient for our SA are reported in Table 5 (first line). The correlation coefficients do not seem to provide any particular insight in the example we consider here.

When the relationship between the input and the output is non-linear, but monotonic, rank transformations may provide interesting insights into inputoutput relationship. Rank transformation consists in replacing the values of the parameters and output by their respective ranks in the data set of values. Hence, any non-linear monotonic relationship becomes a straight line. The computation of correlation coefficients for these rank-transformed parameters and outputs yields the so-called Rank Correlation Coefficient (RCC), that is reported in Table 5 (second line). Those parameters are more interesting here as there is clear evidence of correlation between $k_{A}$ (positive correlation) and $k_{B}$ (negative correlation) and the equilibrium diameter. On the other hand, these coefficients confirm the lack of correlation between $n_{f}$ or $D_{P}$ and the equilibrium diameter.

Finally, we perform an analysis of variance on this model, through the computation of Sobol Indices (Sobol, 2001). These indices predict the influ- 
Table 5: Correlations, rank-correlations, and sensitivity indices of the equilibrium diameter $D_{\infty}$ with respect to flocculation parameters, considering the parameterization with nondimensional $k_{B}$ (upper half of the table) or with dimensional $k_{B}^{\prime}$ (lower half of the table). The second-order Sobol indices that are not indicated are close to zero.

\begin{tabular}{|c|c|c|c|c|}
\hline & $k_{A}$ & $k_{B}$ & $n_{f}$ & $D_{P}$ \\
\hline \hline Pearson Correlation Coefficients (CC) & 0.02 & -0.02 & -0.00 & 0.02 \\
Rank Correlation Coefficient (RCC) & 0.61 & -0.74 & -0.03 & 0.00 \\
Sobol First-Order Sensitivity Indices (SI) & 0.00 & 0.60 & 0.00 & 0.00 \\
Sobol Second-Order Sensitivity Indices & $k_{A}-k_{B}: 0.36$ & - & - \\
Sobol Third-Order Sensitivity Indices & 0.60 & 0.00 & 0.97 & 0.97 \\
\hline \hline Correlation Coefficients (CC) & 0.00 & 0.00 & -0.00 & -0.00 \\
Rank Correlation Coefficient (RCC) & 0.12 & -0.19 & -0.93 & 0.08 \\
Sobol First-Order Sensitivity Indices (SI) & 0.00 & 0.01 & 0.00 & 0.00 \\
Sobol Second-Order Sensitivity Indices & - & $k_{B}^{\prime}-n_{f}:$ & 0.45 & - \\
Sobol Third-Order Sensitivity Indices & 0.03 & 0.00 & 0.03 & 0.64 \\
\hline \hline
\end{tabular}


ence of the variance of each of the input parameters onto the variance of the output, either alone (first-order Sobol indices) or in combinations (higherorder Sobol indices). These indices are listed in Table 5. They indicate that the most influential parameter for the variance of the output is $k_{B}$ (highest first-order Sobol index and lowest third-order Sobol index). The parameter $k_{A}$ is also influential, but only through its influence coupled together with $k_{B}$. Note that, contrarily to the case of independent parameters, the Sobol indices do not sum to one when input parameters are correlated (as is the case here). Note also that third-order indices in Table 5 are reported in the columns of the one parameter (out of the four) which is not considered for the computation of that particular index. Hence, vanishing values in a column indicate the most influential parameters.

\subsection{Choice of parameterization}

As discussed in the introduction, one of the main interests of SA lies in its ability to potentially help in the choice of a parameterization in the view of experimental identification. We therefore complete the above sensitivity study with the corresponding analysis based on the alternative parameterization based on the dimensional breakup function $k_{B}^{\prime}$. The first-order marginal density of $D_{P}$ is presented in Figure 2(b), the sensitivity indices and correlation coefficients are presented in the lower part of Table 5, and the second-order marginal densities are presented in Figure 4. Both the firstand second-order marginal densities appear as more complex than the counterparts obtained with the parameterization with the non-dimensional $k_{B}$. This suggests that the influence is more widely spread over the different pa-

rameters. It is confirmed by the values of the coefficients in Table 5. No 

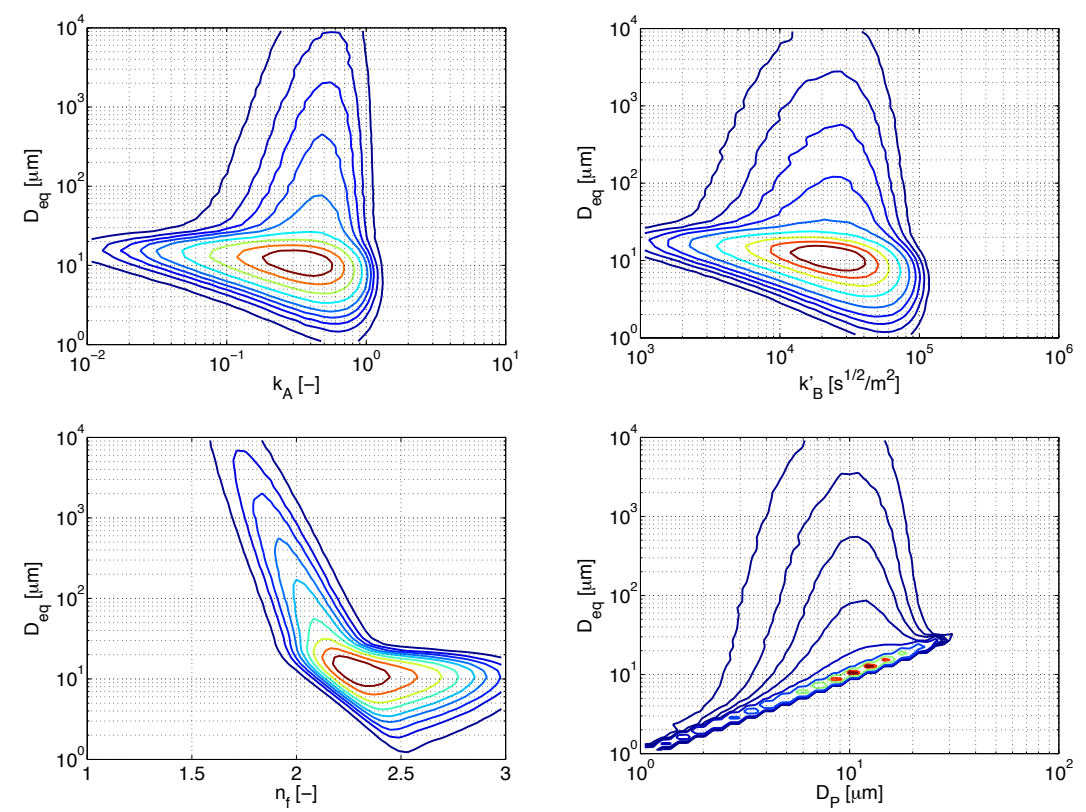

Figure 4: Second-order marginal densities of equilibrium diameter $D_{\infty}$ (considering the parameterization with dimensional $k_{B}^{\prime}$ ) with each of the input parameters. The contour lines correspond to the successive $10 \%$ quantiles. 
parameter is notably correlated with $D_{\infty}$, while the rank correlation coefficients are non-zero for all the parameters. In terms of variance analysis, It is noticeable that three of them have third-order parameters close to zero.

\subsection{Discussion}

The differences observed between the dimensional and non-dimensional approaches are the following: (i) the general structure of the second-order marginal densities to the tensorization of first-order marginal densities in the non-dimensional case, (ii) fewer parameters are correlated significantly with the equilibrium diameter in the non-dimensional case, and (iii) fewer parameters have influence on the variance of the equilibrium diameter in the non-dimensional case. All these (in particular the last point) mean that the identification of $k_{B}$ (or $k_{A}$ to a lesser extent) could be well constrained using equilibrium diameter measures and the non-dimensional model. For this identification procedure, and as they are very little influential, it also means that the values of $n_{f}$ and $D_{P}$ could be chosen quite freely. As the situation is not so simple with the dimensional model, the identification process would have to involve more variables (although $D_{P}$ could probably be left aside) and hence become more difficult. Finally, it should be noted that in the case of non-dimensional models, the recent improved model of the fractal dimension (see Maerz et al. (2011)) seem unnecessary as $n_{f}$ has little influence on this particular QoI. This will be discussed further in Section 5.

\subsection{Additional correlation between fractal dimension and flocculation param- eters}


The Maximum Entropy approach used in Section 3.2 indicates that the rational choice when there is no evidence of dependency between random variables is independence. In order to give more weight to the conclusions of this paper, we nevertheless investigate in this section the possibility that $n_{f}$ be correlated to the other flocculation parameters. To do this, we modify the correlation matrix in Eq. (15) by adding a correlation coefficient $R_{A n}=-0.5$ between $n_{f}$ and $k_{A}$ (both in the dimensional and non-dimensional cases) and correlations coefficient $R_{B n}=R_{B^{\prime} n}=0.5$ between $n_{f}$ and $k_{B}$ and $n_{f}$ and $k_{B}^{\prime}$, respectively. We have no argument for choosing these values rather than others, except that they verify the expected sign. Their amplitude is also large enough to test our hypothesis that the conclusions of the paper still hold when correlation with the fractal dimension is considered. The correlation and sensitivity indices in both these cases are reported in Table 6. Although the indices in the dimensional case seem harder to analyze than without correlation, this is not so in the non-dimensional case, where results are not significantly modified. This suggests that the conclusions we put forward in this paper are really due to the simplicity of the parameter-QoI relationship in the non-dimensional case rather than some particular case of independence of parameters.

\section{Sensitivity analysis in a tidal forcing experiment}

We now turn to a more advanced example of tidal flocculation, already described in Maerz et al. (2011), with comparison with experiments. In this example, the evolution of the diameter of flocs in a turbulence-controlled 0D system under cyclic loading is observed. The loading parameter $G$ follows 
Table 6: Correlations, rank-correlations, and sensitivity indices of the equilibrium diameter $D_{\infty}$ with respect to flocculation parameters considering dependent fractal dimension $n_{f}$, and considering the parameterization with non-dimensional $k_{B}$ (upper half of the table) or with dimensional $k_{B}^{\prime}$ (lower half of the table). The second-order Sobol indices that are not indicated are close to zero.

\begin{tabular}{|c|c|c|c|c|}
\hline & $k_{A}$ & $k_{B}$ & $n_{f}$ & $D_{P}$ \\
\hline \hline Pearson Correlation Coefficients (CC) & 0.02 & -0.02 & -0.04 & 0.02 \\
Rank Correlation Coefficient (RCC) & 0.62 & -0.74 & -0.74 & 0.00 \\
Sobol First-Order Sensitivity Indices (SI) & 0.00 & 0.63 & 0.00 & 0.00 \\
Sobol Second-Order Sensitivity Indices & $k_{A}-k_{B}: 0.38$ & - & - \\
Sobol Third-Order Sensitivity Indices & 0.63 & 0.00 & 1.00 & 1.00 \\
\hline \hline Correlation Coefficients (CC) & 0.00 & -0.00 & -0.00 & -0.00 \\
Rank Correlation Coefficient (RCC) & 0.50 & -0.60 & -0.96 & 0.12 \\
Sobol First-Order Sensitivity Indices (SI) & 0.00 & 0.00 & 0.00 & 0.00 \\
Sobol Second-Order Sensitivity Indices & - & $k_{B}^{\prime} n_{f}: 5.84$ & - \\
Sobol Third-Order Sensitivity Indices & 0.03 & 0.00 & 0.03 & 0.64 \\
\hline \hline
\end{tabular}


a cycle that reproduces what is observed in coastal waters during a tidal cycle. It is varied from $0 \mathrm{~s}^{-1}$ to $12 \mathrm{~s}^{-1}$ on a six-hour time-scale, and is shown in Figure 5. A 0D version (with $|\mathbf{w}|=\left|\mathbf{w}_{s}\right|=0$ ) of Eq. (8) is solved, with a constant mass concentration $c=93 \mathrm{mg} / \mathrm{L}$, to obtain the evolution of the volume concentration $N$ as a function of time. Eq. (1) then provides the evolution of the diameter of the flocs as a function of time. The other numerical parameters are $F_{y}=10^{-10} \mathrm{~kg} \cdot \mathrm{m} / \mathrm{s}^{2}, \mu=10^{-3} \mathrm{~kg} / \mathrm{m} / \mathrm{s}$ and $\rho_{s}=$ $2650 \mathrm{~kg} / \mathrm{m}^{3}$. The initial diameter is equal to the equilibrium diameter $D_{\infty}$ corresponding to the initial turbulence parameter $G=1 \mathrm{~s}^{-1}$.
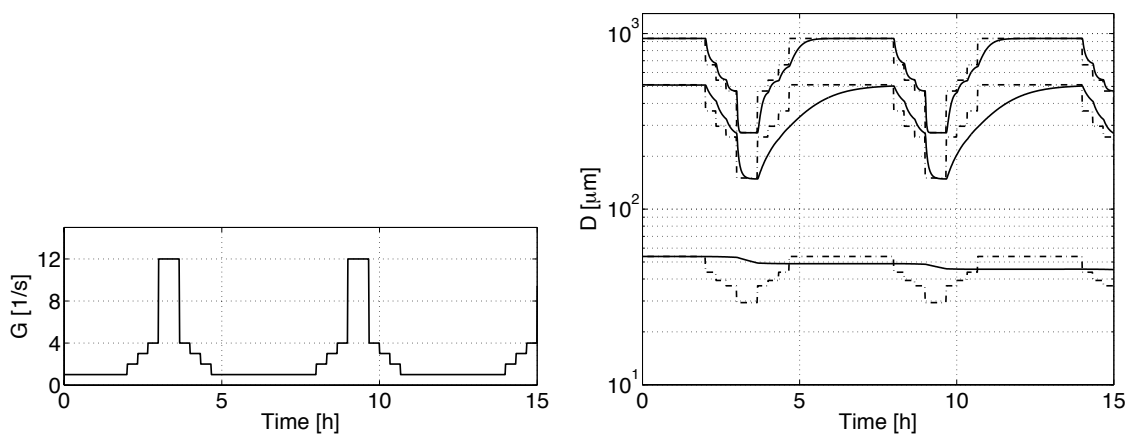

Figure 5: Tidal forcing experiment: (upper figure) evolution of the forcing turbulence parameter; and (lower figure) evolution of the floc diameters (solid lines) and equilibrium diameters (dash-dotted lines) as a function of time, for three realizations of the input parameters.

A previous (local) SA performed on this example (Maerz et al., 2011) stated the fractal dimension as the most influential parameter of the flocculation model for the average diameter. The authors used a model with a dimensional floc-breakup parameter $k_{B}^{\prime}$. This statement is therefore coherent with the results of the (global) analysis performed in the previous section. 
The objective of this section is to check whether the statement is conserved when we consider the flocculation model with a non-dimensional parameter $k_{B}$. This statement has important implications because it is one of the reasons why several authors proposed to refine the modeling of the fractal dimension and consider it to depend on diameter.

We therefore consider in the following the flocculation model with nondimensional breakup parameter $k_{B}$. We then perform a SA with respect to the amplitude of the fluctuations of the diameter, the difference between the minimum and maximum diameters over one tidal cycle, namely over the pe$\operatorname{riod} T=[9-15] h$. We use the Monte Carlo approach, with $10^{5}$ samples and approximate the solution of Eq. (8) for each sample using a Crank-Nicholson scheme for the time discretization. Note that, because of the inertia of the system, these extremal diameters are not equal to the equilibrium diameters corresponding to $G=12 \mathrm{~s}^{-1}$ and $G=1 \mathrm{~s}^{-1}$, respectively. Indeed, the differences in the dynamic behavior of three different samples of the input parameters can be observed on Figure 5. For some samples, the evolution of the diameter (solid lines) follows very closely the evolution of the equilibrium diameter (dashed-dotted lines), while for other samples, the diameter is almost constant throughout. This is related to the value of the equilibrium time $T_{\infty}$, defined in $\mathrm{Eq}(11)$.

We plot on Figure 6 the first-order marginal densities of the minimum and maximum densities, as well as that of the amplitude of fluctuations. On Figure 7 , the second-order marginal densities for the amplitude of fluctuations with respect to the input parameters are plotted. Although they are not as simple as in the previous example (in particular, one appears bi-modal), the 
general shapes of the second-order marginal densities do not seem to show any kind of prominence of the fractal dimension with respect to the other parameters. This is confirmed by the correlation and sensitivity indices reported in Table 7 . The fractal dimension $n_{f}$ does appear in the sensitivity indices, but only in conjunction with the parameter $k_{B}$. The QoI is exactly as sensitive to $n_{f}$ as it is to $k_{A}$, and certainly less than $k_{B}$. The diameter of primary particles is still unimportant for the variance of the output.

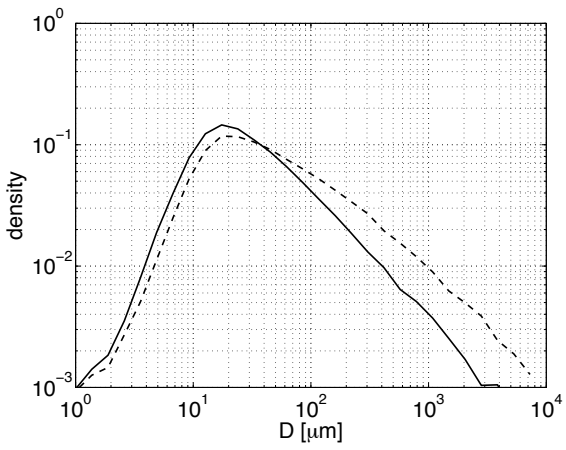

(a) extreme diameters $D_{\min }, D_{\max }$

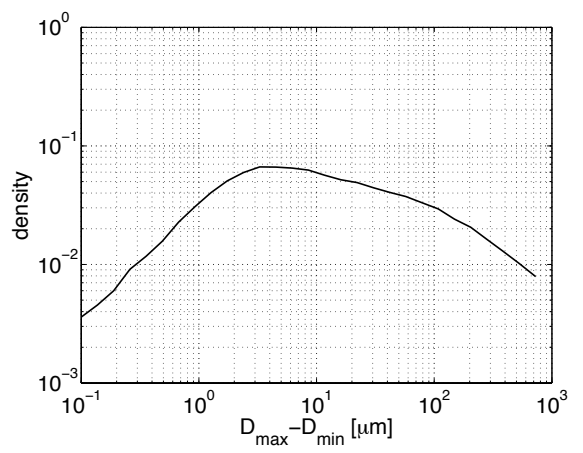

(b) amplitude of fluctuation $D_{\max }-$ $D_{\min }$

Figure 6: Tidal forcing experiment: first-order marginal distributions of minimum and maximum diameters (left figure, solid and dashed lines, respectively), and of the amplitude of fluctuations $D_{\max }-D_{\min }$ (right figure).

We now turn back to the conclusions of the (local) SA in Maerz et al. (2011). When using the flocculation model with dimensional floc-breakup parameter (as Maerz et al. (2011) do), it seems reasonable to refine the modeling of the fractal dimension parameter. The conclusions of our previous global SA (although for a different QoI) seem to confirm the importance of the fractal dimension observed by Maerz et al. (2011). However, the analysis also 

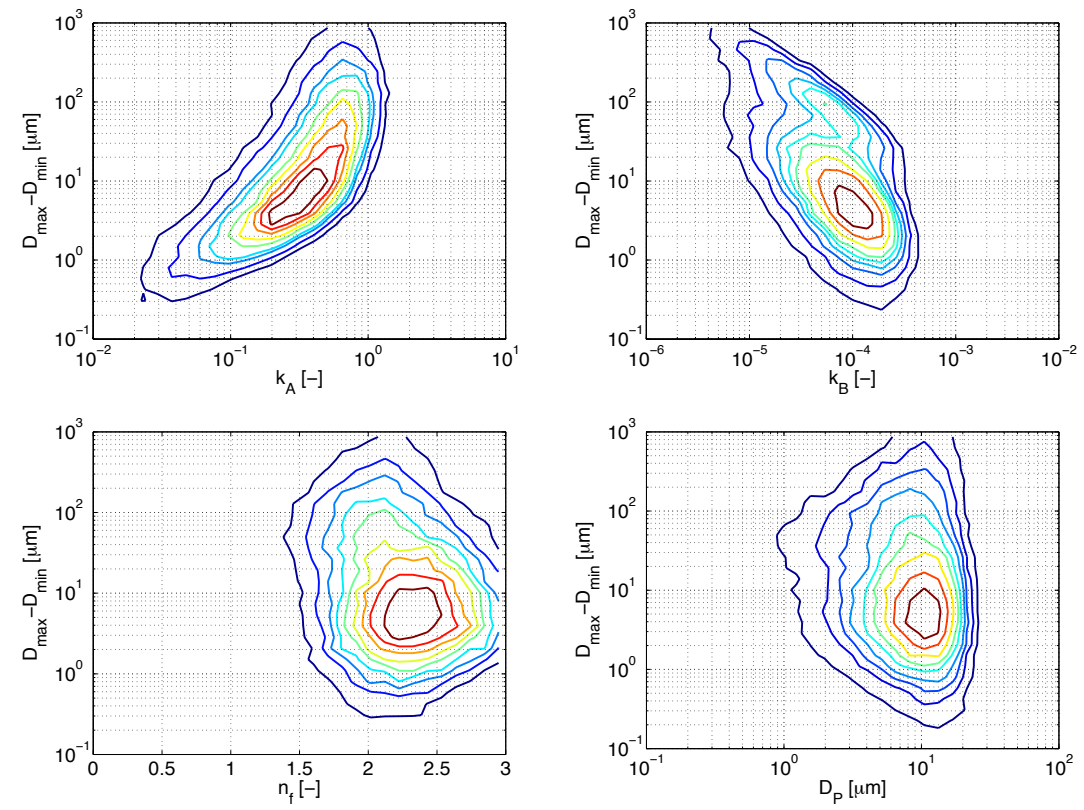

Figure 7: Tidal forcing experiment: second-order marginal densities of the amplitude of fluctuations $D_{\max }-D_{\min }$. The contour lines correspond to the successive $10 \%$ quantiles. 
Table 7: Correlations, rank-correlations, and sensitivity indices of the amplitude of fluctuations $D_{\max }-D_{\min }$. The second-order Sobol indices that are not indicated are close to \begin{tabular}{|c|c|c|c|c|}
\hline zero. & $k_{A}$ & $k_{B}$ & $n_{f}$ & $D_{P}$ \\
\hline \hline Correlation Coefficients (CC) & 0.01 & -0.01 & 0.01 & 0.00 \\
Rank Correlation Coefficient (RCC) & 0.65 & -0.54 & -0.07 & -0.10 \\
Sobol First-Order Sensitivity Indices (SI) & 0.00 & 0.18 & 0.00 & 0.00 \\
Sobol Second-Order Sensitivity Indices & $k_{A^{-}} k_{B}: 0.32$ & - & - \\
& - & $k_{B^{-}} n_{f}: 0.34$ & - \\
Sobol Third-Order Sensitivity Indices & 0.45 & 0.00 & 0.57 & 1.25 \\
\hline
\end{tabular}

seems to suggest that using the flocculation model with a non-dimensional parameter attenuates strongly the influence of the fractal dimension. In that case, the fractal dimension would not need to be modeled more precisely, as Maerz et al. (2011) and others do, for instance by considering it to be a function of floc diameter. As it is much simpler to use the model based on a non-dimensional parameter rather than to make the fractal dimension dependent on the floc diameter, it is advisable to follow the former approach.

\section{Conclusion}

We have reported in this paper a global SA for two different parameterizations of a fractal-based turbulence-induced flocculation model. Using the equilibrium diameter as first QoI, we have shown that the two parameterizations yielded very different results in terms of parameter-QoI correlations and 
sensitivities. Namely, the parameterization in terms of a non-dimensional floc breakup parameter showed strong sensitivity with respect to two parameters only (floc breakup and aggregation parameters), while the parameterization in terms of the dimensional floc breakup parameter presented a more widely distributed sensitivity and more complex correlation patterns. The conclusion of this analysis is that the former parameterization is probably much better suited for an identification experiment based on the measure of floc diameter, provided the fractal dimension and diameter of the primary particle can be measured through other experiments. It also indicates that the equilibrium diameter can be approximated very well by a function of only the corresponding two parameters.

A second SA considered a $0 \mathrm{D}$ experiment reproducing a tidal flocculation process, taken from Maerz et al. (2011). A previous SA by these authors on the characteristic diameter suggested that the fractal dimension was extremely important for this experiment. Our analysis observes that this is only true for the parameterization with dimensional floc breakup parameter. Using the non-dimensional parameterization, as recommended by our previous analysis, the diameter fluctuations appears more sensitive to the floc breakup parameters. The results of Maerz et al. (2011) motivated in particular the introduction of more complex models of the fractal dimension, varying with the diameter. Our analysis seems to indicate that this might be unnecessary, provided that the appropriate parameterization of the model is chosen.

Finally, it should be noted that other QoIs could be considered, and might lead to different conclusions. In particular, Verney et al. (2011) report local 
sensitivity analyses focused on the settling velocity that seem to indicate that the primary diameter $D_{P}$ might be more important for this QoI than in the $\mathrm{SA}$ reported here.

\section{Appendix A. Generation of samples of flocculation parameters}

We describe here a method for generating samples of the flocculation parameters with the chosen first-order marginal distributions and correlation. We consider a generic random vector of dimension 4 , with given first-order marginal distributions for each of its coordinates, and a given correlation matrix. The cumulative distributions are denoted $\Phi_{i}$, for $1 \leq i \leq 4$, and the correlation matrix is denoted $R$.

We start with 4 independent unit centered gaussian random variables $G_{i}$ grouped in a vector $G$, and a correlation matrix $R^{*}$ (the value of which will be discussed below). We then construct the 4-dimensional correlated random

vector $U=G \sqrt{R^{*}}$, where $\sqrt{R^{*}}$ comes from the Choleski decomposition of $R^{*}$. Finally, we transform each of the random variables in the vector $U$ into the appropriate first-order marginal distribution using the classical transformation $K_{i}=\Phi_{i}^{-1}\left(\Phi_{0}\left(U_{i}\right)\right)$, where $\Phi_{0}$ is the cumulative density function of a unit centered gaussian random variable. By doing this last transformation, we unfortunately modify the correlation structure of $U$ (which was by definition $R^{*}$ ) into something else.

The proposed approach is therefore iterative. We start from an initial guess of $R^{*}$ (the obvious choice being $R^{*}=R$ ), compute numerically the resulting correlation for $K$ and iterate until that correlation is close to $R$.

Using this approach and the statistical parameters and laws discussed in 
Section 3.2, we converged to the following correlation matrices:

- for the non-dimensional model (and $n_{f}$ independent of the other parameters)

$$
R^{*}=\left[\begin{array}{cccc}
1 & 0 & 0 & -0.44 \\
0 & 1.08 & 0 & -0.35 \\
0 & 0 & 1 & 0 \\
-0.44 & -0.55 & 0 & 1
\end{array}\right]
$$

- for the dimensional model (and $n_{f}$ independent of the other parameters)

$$
R^{*}=\left[\begin{array}{cccc}
1 & 0 & 0 & -0.44 \\
0 & 1.08 & 0 & -0.55 \\
0 & 0 & 1 & 0 \\
-0.44 & -0.55 & 0 & 1
\end{array}\right]
$$

- for the non-dimensional model (and $n_{f}$ dependent on the other parameters)

$$
R^{*}=\left[\begin{array}{cccc}
1 & 0 & -0.52 & -0.44 \\
0 & 1.08 & 0.57 & -0.35 \\
-0.52 & 0.57 & 1 & 0 \\
-0.44 & -0.35 & 0 & 1
\end{array}\right]
$$

- for the dimensional model (and $n_{f}$ dependent on the other parameters)

$$
R^{*}=\left[\begin{array}{cccc}
1 & 0 & -0.53 & -0.44 \\
0 & 1.08 & 0.55 & -0.53 \\
-0.53 & 0.55 & 1 & 0 \\
-0.44 & -0.53 & 0 & 1
\end{array}\right]
$$


Using these matrices to correlate the gaussian germs allows to generate samples of the parameters following the chosen first-order marginal laws as well as having the right correlation structure.

\section{Acknowledgements}

The authors would like to thank the support of PETROBRAS Technological Program on Basin Modeling in the name of its general coordinator, Dr. Marco Moraes. We also acknowledge the fruitful discussions within the program with Dr. Paulo Paraizo and Prof. Ben Kneller. Partial support was also provided by $\mathrm{MCT} / \mathrm{CNPq}$ and FAPERJ.

Adhikari, S., Friswell, M.I., 2007. Random matrix eigenvalue problems in structural dynamics. Int. J. Num. Meths. Engng. 69, 562-591. doi:10.1002/nme.1781.

Berry, R.D., Najm, H.N., Debusschere, B.J., Marzouk, Y.M., Adalsteinsson, H., 2012. Data-free inference of the joint distribution of uncertain model parameters. J. Comp. Phys. 231, 2180-2198. doi:10.1016/j.jcp.2011.10.031.

Biggs, C.A., Lant, P.A., 2000. Activated sludge flocculation: on-line determination of floc size and the effect of shear. Water Res. 34, 2542-2550. doi:10.1016/S0043-1354(99)00431-5.

Bouyer, D., Liné, A., Do-Quang, Z., 2004. Experimental analysis of floc size distribution under different hydrodynamics in a mixing tank. AIChE J. 50, 2064-2081. doi:10.1002/aic.10242. 
Bushell, G.C., Yan, Y.D., Woodfield, D., Raper, J., Amal, R., 2002. On techniques for the measurement of the mass fractal dimension of aggregates. Adv. Colloid Interface Sci. 95, 1-50. doi:10.1016/S0001-8686(00)00078-6.

Cantero, M.I., Balachandar, S., Garcia, M.H., Ferry, J.P., 2006. Direct numerical simulation of planar and cylindrical density currents. J. Appl. Mech. 73, 923-930.

Chao, X., Jia, Y., 2011. Three-dimensional numerical simulation of cohesive sediment transport in natural lakes, in: Ginsberg, S.S. (Ed.), Sediment transport, InTech.

Cottereau, R., Clouteau, D., Soize, C., 2007. Construction of a probabilistic model for impedance matrices. Comp. Meths. Appl. Mech. Engng. 196, 2252-2268. doi:10.1016/j.cma.2006.12.001.

Cuthbertson, A.J., Dong, P., Davies, P.A., 2010. Non-equilibrium flocculation characteristics of fine-grained sediments in grid-generated turbulent flows. Coastal Engng. 57, 447-460. doi:10.1016/j.coastaleng.2009.11.011.

Elias, R.N., Paraizo, P.L., Coutinho, A.L., 2008. Stabilized edge-based finite element computation of gravity currents in lock-exchange configurations. Int. J. Num. Meths. Engng. 57, 1137-1152. doi:10.1002/fld.1781.

Espath, L.F., Pinto, L.C., Laizet, S., Silvestrini, J.H., 2014. Two- and three-dimensional Direct Numerical Simulation of particle-laden gravity currents. Comp. \& Geosci. 63, 9-16. doi:10.1016/j.cageo.2013.10.006.

Friedlander, S., 1957. Mass and heat transfer to single spheres and cylinders at low reynolds numbers. AIChE J. 3, 43-48. 
Geyer, W.R., Hill, P.S., Kineke, G.C., 2004. The transport, transformation and dispersal of sediment by buoyant coastal flows. Cont. Shelf Res. 24, 927-949. doi:0.1016/j.csr.2004.02.006.

Guerra, G.M., Zio, S., Camata, J.J., Rochinha, F.A., Elias, R.N., Paraizo, P.L., Coutinho, A.L., 2013. Numerical simulation of particle-laden flows by the residual-based variational multiscale method. Int. J. Num. Meth. Fluids 73, 729-749. doi:10.1002/fld.3820.

Helton, J.C., Johnson, J.D., Sallaberry, C.J., Storlie, C.B., 2006. Survey of sampling-based methods for uncertainty and sensitivity analysis. Reliability Engng. Syst. Safety 91, 1175-1209. doi:10.1016/j.ress.2005.11.017.

Hiester, H.R., Piggott, M.D., Allison, P.A., 2011. The impact of mesh adaptivity on the gravity current front speed in a two-dimensional lockexchange. Ocean Modelling 38, 1-21.

Hsu, T.J., Traykovski, P.A., Kineke, G.C., 2007. On modeling boundary layer and gravity-driven fluid mud transport. J. Geophys. Res. 112, C04011. doi:10.1029/2006JC003719.

Jarvis, P., Jefferson, B., Gregory, J., Parsons, S.A., 2005. A review of floc strength and breakage. Water Res. 39, 3121-3137. doi:10.1016/j.watres.2005.05.022.

Jaynes, E.T., 1957. Information theory and statistical mechanics. Phy. Rev. $106,620-630$.

Khelifa, A., Hill, P.S., 2006. Models for effective density and 
settling velocity of flocs. J. Hydraulic Res. 44, 390-401. doi:10.1080/00221686.2006.9521690.

Kranenburg, C., 1994. The fractal structure of cohesive sediment aggregates. Estuarine Coastal Shelf Sci. 39, 451-460. doi:10.1016/S0272$7714(06) 80002-8$.

Levich, V.G., 1962. Physicochemical hydrodynamics. Prentice Hall, Inc.

Lick, W., Huang, H., Jepsen, R., 1993. Flocculation of fine-grained sediments due to differential settling. J. Geophys. Res. 98, 279-288. doi:10.1029/93JC00519.

Maerz, J., Verney, R., Wirtz, K., Feudel, U., 2011. Modeling flocculation processes: intercomparison of a size class-based model and a distributionbased model. Cont. Shelf Res. 31, S84-S93. doi:10.1016/j.csr.2010.05.011.

Maggi, F., Winterwerp, J.C., 2004. Method for computing the threedimensional capacity dimension from two-dimensional projections of fractal aggregates. Phys. Rev. E 69, 011405. doi:10.1103/PhysRevE.69.011405.

Maggi, F., Winterwerp, J.C., Fontijn, H.L., van Kestern, W.G., Cornelisse, J.M., 2002. A settling column for turbulence-induced flocculation of cohesive sediments, in: Wahl, T.L., Pugh, C.A., Oberg, K.A., Vermeyen, T.B. (Eds.), Proceedings of HMEM2002 Conference, Estes Park, Colorado, USA. p. 93. doi:10.1061/40655(2002)34.

Manning, A.J., Dyer, K.R., 1999. A laboratory examination of floc characteristics with regard to turbulent shearing. Marine Geol. 160, 147-170. doi:10.1016/S0025-3227(99)00013-4. 
McAnally, W.H., Mehta, A.J., 2002. Significance of aggregation of fine sediment particles in their deposition. Estuarine Coastal Shelf Sci. 54, 643-653. doi:10.1006/ecss.2001.0847.

Meakin, P., 1984. Effects of cluster trajectories on cluster-cluster aggregation: a comparison of linear and Brownian trajectories in two- and three-dimensional simulations. Phys. Rev. A 29, 997-999. doi:10.1103/PhysRevA.29.997.

Meiburg, E., Kneller, B., 2010. Turbidity currents and their deposits. Annu. Rev. Fluid Mech. 42, 135-156. doi:10.1146/annurev-fluid-121108-145618.

Mietta, F., Maggi, F., Winterwerp, J.C., 2008. Sensitivity to breakup functions of a population balance equation for cohesive sediments. Proc. Marine Sci. 9, 275-286. doi:10.1016/S1568-2692(08)80021-6.

Nasr-Azadani, M.M., Hall, B., Meiburg, E., 2013. Polydisperse turbidity current propagating over complex topography: comparison of experimental and depth-resolved simulation results. Comp. \& Geosci. 53, 141-153. doi:10.1016/j.cageo.2011.08.030.

O'Melia, C., 1980. Aquasols: the behaviour of small particles in acquatic systems. ES\&T 14, 1052-1060.

Rahmani, N.H., Dabros, T., Masliyah, J.H., 2004. Evolution of asphaltene floc size distribution in organic solvents under shear. Chem. Engr. Sci. 59, 685-697. doi:10.1016/j.ces.2003.10.017.

Saltelli, A., Chan, K., Scott, E.M., 2000. Sensitivity analysis: gauging the worth of scientific models. Wiley. 
Saltelli, A., Ratto, M., Andres, T., Campolongo, F., Cariboni, J., Gatelli, D., Salsana, M., Tarantola, S., 2008. Global sensitivity analysis: the primer. John Wiley \& Sons Ltd.

Serra, T., Casamitjana, X., 1998. Structure of the aggregates during the process of aggregation and breakup under a shear flow. J. Colloid Interf. Sci. 206, 505-511. doi:10.1006/jcis.1998.5714.

Shannon, C.E., 1948. A mathematical theory of communication. Bell Syst. Technol. J. 27, 379-423; 623-656.

von Smoluchowski, M., 1917. Versuch einer mathematischen theorie der koagulationskinetik kolloider lösungen. Zeitschrift für Physikalische Shemie 92, 129-168. In German.

Sobol, I.M., 2001. Global sensitivity indices for nonlinear mathematical models and their Monte Carlo estimates. Math. Comp. Simul. 55, 271-280. doi:10.1016/S0378-4754(00)00270-6.

Soize, C., 2000. A nonparametric model of random uncertainties for reduced matrix models in structural dynamics. Prob. Engng. Mech. 15, 277-294. doi:10.1016/S0266-8920(99)00028-4.

Son, M., Hsu, T.J., 2008. Flocculation model of cohesive sediment using variable fractal dimension. Environmental Fluid Mech. 8, 55-71. doi:10.1007/s10652-007-9050-7.

Spicer, P.T., Pratsinis, S.E., 1996. Coagulation and fragmentation: universal steady-state particle-size distribution. AIChE J. 42, 1612-1620. doi:10.1002/aic.690420612. 
Thomas, D.N., Judd, S.J., Fawcett, N., 1999. Flocculation modelling: a review. Water Res. 33, 1579-1592. doi:10.1016/S0043-1354(98)00392-3.

Thouy, R., Jullien, R., 1994. A cluster-cluster aggregation model with tunable fractal dimension. J. Phys. A: Math. Gen. 27, 2953. doi:10.1088/0305$4470 / 27 / 9 / 012$.

Udwadia, F.E., 1989. Some results on maximum entropy distributions for parameters known to lie in finite intervals. SIAM Rev. 31, 103-109.

Vahedi, A., Gorczyca, B., 2011. Application of fractal dimensions to study the structure of flocs formed in lime softening process. Water Res. 45, 545-556. doi:10.1016/j.watres.2010.09.014.

Vahedi, A., Gorczyca, B., 2012. Predicting the settling velocity of flocs formed in water treatment using multiple fractal dimensions. Water Res. 46, 4188-4194. doi:10.1016/j.watres.2012.04.031.

Verney, R., Lafite, R., Brun-Cottan, J.C., Le Hir, P., 2011. Behaviour of a floc population during a tidal cycle: laboratory experiments and numerical modelling. Cont. Shelf Res. 31, S64-S83. doi:10.1016/j.csr.2010.02.005.

Winterwerp, J.C., 1998. A simple model for turbulence induced flocculation of cohesive sediment. J. Hydraulic Res. 36, 309-326. doi:10.1080/00221689809498621.

Xu, F., Wang, D.P., Riemer, N., 2008. Modeling flocculation processes of fine-grained particles using a size-resolved method: comparison with published laboratory experiments. Cont. Shelf Res. 28, 2668-2677. doi:10.1016/j.csr.2008.09.001. 